\title{
Haemolytic anaemia complicating the concurrent use of allopurinol \& azathioprine after kidney transplantation
}

\author{
Catherine Hanna $^{1}{ }^{*}$, Neeraj Dhaun ${ }^{1}$, Maria Squires ${ }^{2}$, Simon Watson ${ }^{1}$
}

\author{
${ }^{1}$ Department of Renal Medicine, \\ ${ }^{2}$ Department of Clinical \\ Biochemistry, Royal Infirmary of \\ Edinburgh, 53 Little France \\ Crescent, Edinburgh. EH16 4SA, \\ UK
}

Received: 16 May 2013

Accepted: 24 May 2013

*Correspondence to:

Dr. Catherine Hanna,

Email: cath.hanna@gmail.com

(C) 2013 Hanna $\mathrm{C}$ et al. This is an open-access article distributed under the terms of the Creative Commons Attribution License, which permits unrestricted use, distribution, and reproduction in any medium, provided the original work is properly cited.

\begin{abstract}
Gout is a common problem in renal transplant recipients but often difficult to treat. Allopurinol can be combined with azathioprine but clinicians should be aware of the need for dose reduction, the potential to measure azathioprine breakdown products and the possible side effects of this combination. Leucopenia is a known side effect but this case report shows that haemolytic anaemia can also occur.
\end{abstract}

Keywords: Allopurinol, Azathioprine, Transplantation, Haemolytic anemia

\section{INTRODUCTION}

Elevated serum uric acid (sUA) is common in kidney transplant recipients. ${ }^{1}$ It is an important risk factor for reduced graft survival ${ }^{2}$ and incident cardiovascular disease $^{3}$ and may lead to the development of the painful arthopathy 'gout'. Importantly, emerging clinical data show that lowering sUA has both cardiovascular and renal benefits ${ }^{4}$, as well as reducing the frequency of gout episodes.

Xanthine oxidase inhibitors such as allopurinol effectively lower sUA. Their use in transplant recipients is limited by their inhibition of the metabolism of the immunosuppressive agent azathioprine. Coadministration of allopurinol and azathioprine leads to accumulation of potentially toxic thiopurine metabolites (TM) which cause leucopenia. ${ }^{5}$ Co-prescription can be done but with care. Here we describe another important but less well-known side effect of concomitant azathioprine and allopurinol use.

\section{CASE REPORT}

A 56 year-old gentleman with end-stage renal failure due to reflux nephropathy received a kidney transplant from a living related donor 15 years earlier. Maintenance immunosuppression was ciclosporin $100 \mathrm{mg}$ twice daily and azathioprine $100 \mathrm{mg}$ daily. He suffered frequent attacks of gout with sUA persistently over $0.42 \mathrm{mmol} / 1$ $(0.12-0.42)$. Allopurinol $100 \mathrm{mg}$ daily was commenced and azathioprine was replaced with mycophenolate mofetil (MMF) to avoid TM accumulation. Unfortunately, MMF was not tolerated due to severe skin rash and mouth ulceration and so allopurinol was stopped to allow re-introduction of azathioprine. Colchicine was used for gout control but this was poorly tolerated due to diarrhoea. In the following two years the patient experienced increasingly disabling gout and eventually allopurinol was re-introduced alongside a $50 \%$ reduction in the dose of azathioprine.

Over the next three months the patient developed symptomatic anaemia with a fall in haemoglobin $(\mathrm{Hb})$ 
from 120 to $87 \mathrm{~g} / 1$ (135 - 180) (Figure 1). There was no evidence of blood loss. Mean corpuscular volume rose from 91 to $107 \mathrm{fl}(78-98)$ and bilirubin from 13 to $37 \mu \mathrm{mol} / 1(3-21)$. Serum vitamin $B_{12}$, folic acid and iron studies were normal and there was no change in the serum creatinine, white cell or platelet counts. Further investigations revealed evidence of haemolysis with low haptoglobins $(<0.1 \mathrm{~g} / \mathrm{L}$, normal $0.2-2.6 \mathrm{~g} / \mathrm{L})$, a reticulocytosis $\left(115 \times 10^{9} / \mathrm{L}\right.$, normal $\left.25-85\right)$, and a rising lactate dehydrogenase concentration (213 U/L, normal 125 - $220 \mathrm{U} / \mathrm{L})$. A blood film showed macrocytosis, polychromasia and anisochromia. A direct Coombs test was negative.

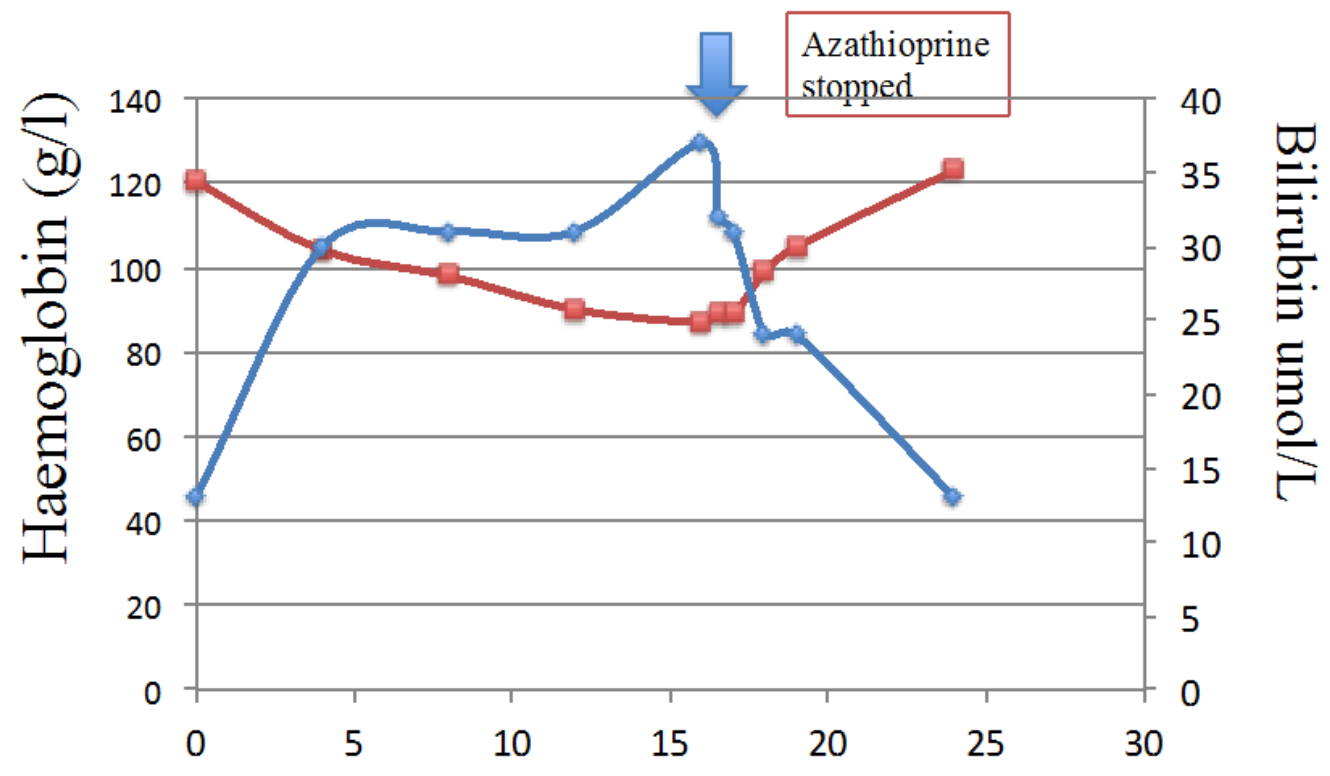

\section{Weeks after allopurinol and azathioprine co-prescription}

Figure 1: Trend in haemoglobin and bilirubin after azathioprine and allopurinol co-prescription.

\section{DISCUSSION}

Azathioprine is an inactive pro-drug and its immune suppressant activity is dependent upon metabolism to 6thioguanine metabolites (6-TGN) by a series of multienzyme processes. Two routes of inactivation limit this: oxidation by xanthine oxidase to 6-thiouric acid and thiol methylation by thiopurine methyltransferase (TPMT) to form 6 methylmercaptopurine (6-MMPN), a metabolite which in high concentrations causes hepatotoxicity. When co-administered, allopurinol inhibits xanthine oxidase activity and leads to the preferential formation of 6-TGN. Intuitively it would be anticipated that 6-MMPN concentrations would also rise, however there is in fact a dramatic reduction in these concentrations, the mechanism of which is unknown. ${ }^{5}$ In accordance with this, measurement of azathioprine metabolites in this patient revealed a high 6-TGN $(844 \mathrm{pmol} / 8 \quad$ x10 erythrocytes, normal 235 - 450) and a low 6-MMPN concentration (<100pmol/8 $\quad$ x $10^{8}$ erythrocytes). Azathioprine was stopped and within one month there was nearly complete normalization of serum markers (Figure 1). Ongoing immunosuppression consisted of prednisolone and ciclosporin only. Allopurinol was continued for gout control.
Transplant clinicians see many cases of gout. Although xanthine oxidase inhibitors can provide excellent control of symptoms an awareness of the potential for toxic interaction with azathioprine is important. We believe it is equally important for clinicians to appreciate that TM toxicity can result in haemolytic anemia ${ }^{6}$, as well as leucopenia.

\section{Funding: No funding sources Conflict of interest: None declared Ethical approval: Not required}

\section{REFERENCES}

1. Stamp L, Ha L, Searle M, O'Donnell J, Frampton C, Chapman P. Gout in renal transplant recipients. Nephrology (Carlton) 2006;11:367-71.

2. Haririan A, Nogueira J M, Zandi-Nejad K, Aiyer R, Hurley H, Cooper M, Klassen D K, Weir M R. The independent association between serum uric acid graft outcomes after kidney transplantation. Transplantation 2010;89:573-9.

3. Feig DI, Kang DH, Johnson RJ. Uric acid and cardiovascular risk. N Engl J Med 2008;359:181121. 
4. Talaat KM, el-Sheikh AR. The effect of mild hyperuricemia on urinary transforming growth factor beta and the progression of chronic kidney disease. Am J Nephrol 2007;27:435-40.

5. Gearry RB, Day AS, Barclay ML, Leong RW, Sparrow MP. Azathioprine and allopurinol: A twoedged interaction. J Gastroenterol Hepatol 2010;25:653-5.
6. Zazgornik J, Hruby K, Schmidt P, Kopsa H, Pils P. Hemolysis after combined azathioprine and allopurinol therapy. Acta Med Austriaca 1979;6:197-9.

doi:10.5455/2319-2003.ijbcp20130619

Cite this article as: Hanna C, Dhaun N, Squires M, Watson S. Haemolytic anaemia complicating the concurrent use of allopurinol \& azathioprine after kidney transplantation. Int J Basic Clin Pharmacol $2013 ; 2: 330-2$. 